heitlich unbedenklich sei das Produkt aber dennoch nicht. Bei der Beurteilung gesundheitlicher Risiken müsse aber immer berücksichtigt werden, womit man vergleicht, und im Falle der Tabakziga- rette schneide die E-Zigarette besser ab. Daher riet der Suchtmediziner, die E-Zigarette nicht voreilig als Option zur Rauchentwöhnung zu verwerfen, nicht zuletzt da laut Stöver nicht übermäßig viele wirksame Alternativen zur Verfügung stehen.

Dr. Dagmar Kraus

Stöver H. E-Zigarette bei Tabakabhängigkeit. 17. Interdisziplinärer Suchtkongress. München, 30.6. - 2.7.2016

\title{
Warum werden junge Männer zu Spielern?
}

Das problematische oder pathologische Glücksspiel ist vorrangig ein Problem junger Männer und oftmals mit Stress und stressauslösenden Ereignissen assoziiert.

$\mathrm{N}$ ach Daten der Bundeszentrale für gesundheitliche Aufklärung ist die Prävalenz der Spielsucht in der Altersgruppe der 21- bis 25-Jährigen gegenüber der Allgemeinbevölkerung etwa dreifach erhöht (2,7\% vs. 0,8\%). Eine mögliche Erklärung liefert die Münchner „Freizeit-Studie“: Nach Pawel Sleczka vom Münchner Institut für Therapieforschung (IFT) ist Glücksspielen mit Stress und stressauslösenden Ereignissen assoziiert. Den Betroffenen mangelt es offenbar an der Fähigkeit zum „funktionellen Handlungsaufschub“, weshalb sie in stressreichen Situationen voreilig agieren.

Im Rahmen der seit Januar 2014 laufenden Studie haben insgesamt 2.693 Personen an einer Querschnittsbefragung teilgenommen. 135 Personen, die sich regelmäßig an Glücksspielen beteiligten oder Glücksspielprobleme aufwiesen, wurden für Folgebefragungen ausgewählt.

Insgesamt spielten 7,4\% der Teilnehmer „häufig“, das heißt mindestens einmal pro Woche. 5,0\% erfüllten mindes-

tens ein und 1,2\% vier oder mehr diagnostische Kriterien für eine Störung durch Glücksspiel gemäß DSM-5. $20 \%$ berichteten, dass sie infolge negativer Lebensereignisse an Glücksspielen teilnahmen. $10 \%$ hatten als Folge des Glücksspiels suizidale Gedanken.

Die Teilnehmer mit problematischem Glücksspiel oder mit einer diagnostizierten Störung durch Glücksspiel wiesen deutlich niedrigere Werte auf der Skala "funktionaler Handlungsaufschub" auf. Es gelang ihnen auch seltener, das Auftreten von Stress zu vermeiden.

Sleczka und sein Team wollen die Problematik über das „proaktive Coping" angehen, das vorausschauende Verhalten zur Vermeidung negativer Zustände. Die Freizeit-Studie habe gezeigt, dass diese Fähigkeit bei den Personen mit einer Störung durch Glücksspiel deutlich verringert sei. Wie erste Ergebnisse nahelegen, ließ sich das präventive Coping im professionellen Setting bei den meisten Glücksspielern

\begin{abstract}
Hilfsangebote für Angehörige
Angehörige pathologischer Glücksspieler sind oft ebenso stark belastet wie die Spieler selbst. Dennoch nehmen die Betroffenen - zu $80 \%$ Frauen - nur selten Hilfe von außen in Anspruch, sei es aus Scham oder aus dem Gefühl heraus, "es alleine schaffen zu können". ETAPPE („Entlastungstraining für Angehörige problematischer und pathologischer Glücksspieler - psychoedukativ") ist ein Hilfsangebot, das den Angehörigen Informationen über die Spielsucht und individuelle Bewältigungsfähigkeiten vermitteln soll. Erste Ergebnisse zeigen eine deutliche Verringerung der subjektiven Belastung. Um möglichst viele Betroffene zu erreichen, wurde zusätzlich das E-Mental-Health-Angebot "Verspiel nicht mein Leben - Entlastung für Angehörige" entwickelt. Die Internetseite www.verspiel-nichtmein-leben.de stellt erste Hilfen bereit und informiert über weitere Angebote.
\end{abstract}

verstärken. Die Forscher sehen darin einen möglichen Ansatz für eine Erweiterung des Behandlungsangebots.

Dr. Elke Oberhofer

17. Interdisziplinärer Kongress für Suchtmedizin, München, 30.6.-2.7.2016

\section{Stimulanzieneinsatz - Gratwanderung zwischen Therapieerfolg und Psychose}

Stimulanzien können einerseits hilfreich sein, weil sie etwa bei ADHSPatienten die kognitiven Fähigkeiten verbessern und die Aufmerksamkeit erhöhen. Da sie ins dopaminerge System eingreifen, das von einer Reihe individueller Faktoren beeinflusst wird, ist ihr Einsatz jedoch nicht ohne Risiko.

$\mathrm{M}$ it dem Einsatz von Stimulanzien zu Therapiezwecken wird bereits seit Jahrzehnten experimentiert. Von der
Struktur her ähnlich wie Dopamin oder Noradrenalin greifen sie an den entsprechenden Orten im Gehirn an und kön- nen so möglicherweise nutzbringend sein - sofern es gelingt, ihre Wirkung unter Kontrolle zu halten. Aber genau darin besteht offenbar das Problem.

Nach Christian Schütz von der University of British Columbia in Vancouver gibt es zahlreiche Studien, die zeigen, dass sich durch Stimulanzien die kognitive Kontrolle und Motivation verbessern lassen. Die bekannteste Indikation: ADHS. Unter Methylphenidat kommt es bei vielen Patienten zu einer Steigerung von Wachsamkeit und Aufmerksamkeit 